01

\title{
Параметры ядерного квадрупольного взаимодействия и пространственное распределение электронных дефектов в решетках $\mathrm{YBa}_{2} \mathrm{Cu}_{3} \mathrm{O}_{7}$ и $\mathrm{La}_{2-x} \mathrm{Sr}_{x} \mathrm{CuO}_{4}$
}

\author{
(С) Е.И. Теруков ${ }^{1,2}$, А.В. Марченко ${ }^{3}$, П.П. Серегин ${ }^{3, \text { ฯ В.С. Киселев }}{ }^{3}$, К.Б. Шахович ${ }^{3}$ \\ ${ }^{1}$ Физико-технический институт им. А.Ф. Иоффре РАН, \\ Санкт-Петербург, Россия \\ ${ }^{2}$ Санкт-Петербургский государственный электротехнический университет „ЛЭТИ“ им. В.И. Ульянова (Ленина), \\ Санкт-Петербург, Россия \\ ${ }^{3}$ Российский государственный педагогический университет им. А.И. Герцена, \\ Санкт-Петербург, Россия \\ ฯ E-mail: ppseregin@mail.ru
}

(Поступила в Редакцию 3 апреля 2018 г.)

На основе сравнения рассчитанных и экспериментальных параметров ядерного квадрупольного взаимодействия в узлах кристаллических решеток сверхпроводящих металлоксидов меди $\mathrm{YBa}_{2} \mathrm{Cu}_{3} \mathrm{O}_{7}$ и $\mathrm{La}_{2-x} \mathrm{Sr}_{x} \mathrm{CuO}_{4}$ показано, что эффективные заряды всех атомов решеток сверхпроводящих керамик соответствуют стандартным степеням их окисления за исключением атомов цепочечного и планарного кислорода в решетке $\mathrm{YBa}_{2} \mathrm{Cu}_{3} \mathrm{O}_{7}$ и атомов планарного кислорода в решетке $\mathrm{La}_{2-x} \mathrm{Sr}_{x} \mathrm{CuO}_{4}$, пониженный заряд которых интерпретируется как результат локализации в соответствующих подрешетках дырки.

DOI: 10.21883/FTT.2018.10.46510.091

\section{1. Введение}

Определение эффективных зарядов атомов в узлах кристаллической решетки позволяет сузить круг допустимых моделей в квантово-механических расчетах электронных свойств высокотемпературных сверхпроводников [1]. Сведения о зарядах атомов могут быть получены из анализа параметров ядерного квадрупольного взаимодействия (ЯКВ), т.е. взаимодействия электрического квадрупольного момента ядра-зонда с градиентом электрического поля (ГЭП) на нем.

Диагонализированный тензор ГЭП на ядрах зонда в кристаллической решетке характеризуется главной компонентой $U_{z z}$ и параметром асимметрии $\eta=\left(U_{x x}-U_{y y}\right) / U_{z z}$, где компоненты тензора ГЭП $U_{x x}$, $U_{y y}, U_{z z}$ связаны уравнением Лапласа $U_{x x}+U_{y y}+U_{z z}=0$ и специальным выбором осей $\left|U_{x x}\right| \leq\left|U_{y y}\right| \leq\left|U_{z z}\right|$. Экспериментальными параметрами, описывающими ЯКВ, являются постоянная квадрупольного взаимодействия $C=e Q U_{z z} \quad$ (здесь $e Q \quad$ квадрупольный момент атома-зонда) и параметр асимметрии $\eta$. В общем случае

$$
\begin{gathered}
e Q U_{z z}=e Q(1-\gamma) V_{z z}+e Q(1-R) W_{z z}, \\
\eta=\left(1 / U_{z z}\right)(1-\gamma) V_{z z} \eta_{\text {lat }}+(1-R) W_{z z} \eta_{\text {val }},
\end{gathered}
$$

где $V_{z z}, W_{z z}, U_{z z}$ - главные компоненты тензоров решеточного ГЭП (он создается ионами решетки), валентного ГЭП (он создается несферическими валентными электронами атома-зонда) и суммарного ГЭП, $\quad \eta_{\text {lat }}=\left(V_{x x}-V_{y y}\right) / V_{z z}, \quad \eta_{\text {val }}=\left(W_{x x}-W_{y y}\right) / W_{z z}$, $\eta$ - параметры асимметрии этих тензоров, $\gamma$ и $R-$ коэффициенты Штернхеймера для зонда, которые учи- тывают антиэкранирование и экранирование внутренними электронными оболочками атома-зонда градиента электрического поля, создаваемого внешними зарядами.

Информация о параметрах ЯКВ в решетках $\mathrm{YBa}_{2} \mathrm{Cu}_{3} \mathrm{O}_{7}$ и $\mathrm{La}_{2-x} \mathrm{Sr}_{x} \mathrm{CuO}_{4}$ была получена методами ядерного магнитного резонанса (ЯМР) и ядерного квадрупольного резонанса (ЯКР) на изотопах ${ }^{17} \mathrm{O}[2,3]$, ${ }^{63} \mathrm{Cu}[3-5],{ }^{137} \mathrm{Ba}[6],{ }^{139} \mathrm{La} \mathrm{[5]} \mathrm{и} \mathrm{эмиссионной} \mathrm{мессбауэ-}$ ровской спектроскопии (ЭМС) [7], а также были сделаны расчеты тензора ГЭП в узлах указанных решеток квантово-механическими методами [8-10]. Однако сравнение расчетных и экспериментальных параметров ЯКВ не дали убедительных результатов по определению эффективных зарядов атомов в указанных соединениях из-за большого числа произвольно выбираемых параметров в квантово-механических расчетах [7].

В этом аспекте следует обратить внимание, что для зондов с полностью (или на половину) заполненной валентной оболочкой $W_{z z} \approx 0$ (такой зонд называется кристаллическим), расчет тензора решеточного ГЭП может быть проведен в рамках модели точечных зарядов, а сопоставление расчетных параметров тензора решеточного ГЭП $\left(V_{z z}, \eta_{\text {lat }}\right)$ экспериментальных параметров ЯКВ

$$
C_{\exp }=\alpha V_{z z}, \quad \eta_{\exp }=\left(V_{x x}-V_{y y}\right) / V_{z z}
$$

где $\alpha=e Q(1-\gamma)$ позволяет определить эффективные заряды всех атомов решетки.

При исследовании $\mathrm{YBa}_{2} \mathrm{Cu}_{3} \mathrm{O}_{7}$ и $\mathrm{La}_{2-x} \mathrm{Sr}_{x} \mathrm{CuO}_{4}$ методом ЭМС условия кристалличности зондов выполняются для изотопов ${ }^{67} \mathrm{Cu}\left({ }^{67} \mathrm{Zn}\right)$ и ${ }^{67} \mathrm{Ga}\left({ }^{67} \mathrm{Zn}\right)[11,12]$. В процессе легирования атомы ${ }^{67} \mathrm{Cu}$ занимают узлы меди решеток $\mathrm{YBa}_{2} \mathrm{Cu}_{3} \mathrm{O}_{7}$ и $\mathrm{La}_{2-x} \mathrm{Sr}_{x} \mathrm{CuO}_{4}$, тогда как 
вследствие близости химических свойств галлия, иттрия и лантана атомы ${ }^{67} \mathrm{Ga}$ изовалентно замещают атомы $\mathrm{Y}$ и La в указанных решетках. После распада материнских ядер ${ }^{67} \mathrm{Cu}$ и ${ }^{67} \mathrm{Ga}$, введенных в указанные решетки, в узлах меди, иттрия или лантана оказывается кристаллический зонд ${ }^{67} \mathrm{Zn}^{2+}$. Этот зонд имеет сферически симметричную $d$-оболочку, а время жизни мессбауэровского уровня ${ }^{67} \mathrm{Zn}$ составляет $\sim 10^{-5} \mathrm{~s}$, что недостаточно для образования дефектов, компенсирующих возможную разницу зарядов мессбауэровского зонда и замещаемого иона. Таким образом, окружение примесных атомов цинка оказывается таким же, как у замещаемых атомов, и это позволяет определить параметры тензора решеточного ГЭП, создаваемого в узлах меди, иттрия и лантана ионами кристаллической решетки.

Как следует из соотношений (2), сравнение расчетных $V_{z z}$ и экспериментальных величин $C_{\exp }$ возможно, если известны величины квадрупольного момента ядра $Q$ и коэффициента Штернхеймера $\gamma$ атома-зонда. Авторы $[11,12]$ для определения эффективных зарядов атомов в решетках $\mathrm{YBa}_{2} \mathrm{Cu}_{3} \mathrm{O}_{7}$ и $\mathrm{La}_{2-x} \mathrm{Sr}_{x} \mathrm{CuO}_{4}$ провели сравнение отношений постоянных квадрупольного взаимодействия для пар одинаковых атомов в различных кристаллографических позициях $P_{l m}=C_{l} / C_{m}$ и отношений главных компонент тензоров ГЭП для тех же пар $p_{l m}=V_{z z l} / V_{z z m}$. Предполагалось, что значения $P_{l m}$ не зависят от коэффициентов Штернхеймера и квадрупольного момента ядра, поскольку относятся к одному и тому же зонду. Однако эффективные заряды атомов в этом случае были определены лишь в единицах заряда атомов иттрия (или лантана), что, естественно, снижает ценность информации.

В настоящей работе пространственное распределение электронных дефектов в решетках $\mathrm{YBa}_{2} \mathrm{Cu}_{3} \mathrm{O}_{7}$ и $\mathrm{La}_{2-x} \mathrm{Sr}_{x} \mathrm{CuO}_{4}$ определено путем сравнения рассчитанных (в рамках модели точечных зарядов) и экспериментальных параметров ЯКВ с использованием экспериментально определенных величин $\alpha_{\mathrm{O}}=e Q_{\mathrm{O}}\left(1-\gamma_{\mathrm{O}}\right)$ и $\alpha_{\mathrm{Zn}}=e Q_{\mathrm{Zn}}\left(1-\gamma_{\mathrm{Zn}}\right)$ для зондов ${ }^{17} \mathrm{O}^{2-}$ и ${ }^{67} \mathrm{Zn}^{2+}$ соответственно (здесь $Q_{\mathrm{O}}$ и $Q_{\mathrm{Zn}}-$ квадрупольные моменты ядер ${ }^{17} \mathrm{O}$ и ${ }^{67} \mathrm{Zn}, \gamma_{\mathrm{O}}$ и $\gamma_{\mathrm{Zn}}-$ коэффициенты Штернхеймера для кристаллических зондов $\mathrm{O}^{2-}$ и $\mathrm{Zn}^{2+}$ ). Поскольку для определения эффективных зарядов всех атомов решеток $\mathrm{YBa}_{2} \mathrm{Cu}_{3} \mathrm{O}_{7}$ и $\mathrm{La}_{2-x} \mathrm{Sr}_{x} \mathrm{CuO}_{4}$ данных ЭМС на изотопах ${ }^{67} \mathrm{Cu}\left({ }^{67} \mathrm{Zn}\right)$ и ${ }^{67} \mathrm{Ga}\left({ }^{67} \mathrm{Zn}\right)$ оказывается недостаточным (поскольку число измеряемых параметров меньше числа определяемых величин), то были привлечены также данные ЯМР на изотопе ${ }^{17} \mathrm{O}[2,3]$. Подтверждение предложенных моделей пространственного распределения электронных дефектов в решетке соединения $\mathrm{YBa}_{2} \mathrm{Cu}_{3} \mathrm{O}_{7}$ получено в результате анализа данных ЯКР на изотопе ${ }^{137} \mathrm{Ba}[6]$, а в решетках твердых растворов $\mathrm{La}_{2-x} \mathrm{Sr}_{x} \mathrm{CuO}_{4}(x=0.1-1.0)$ - в результате анализа параметров ЯКВ в катионных узлах с использованием данных ЭМС на изотопах ${ }^{5} 7 \mathrm{Co}\left({ }^{57 m} \mathrm{Fe}\right)$ и ${ }^{155} \mathrm{Eu}\left({ }^{155} \mathrm{Gd}\right)$.

\section{2. Методика эксперимента}

2.1. Расчет тензоров ГЭП. В качестве объектов исследования были выбраны керамики состава $\mathrm{YBa}_{2} \mathrm{Cu}_{3} \mathrm{O}_{7}, \mathrm{YBa}_{2} \mathrm{Cu}_{3} \mathrm{O}_{6}, \mathrm{Cu} 2 \mathrm{O}$ и $\mathrm{La}_{2-x} \mathrm{Sr}_{x} \mathrm{CuO}_{4}$. Соединение $\mathrm{YBa}_{2} \mathrm{Cu}_{3} \mathrm{O}_{7}$ имеет орторомбическую структуру [13], соединение $\mathrm{RBa}_{2} \mathrm{Cu}_{3} \mathrm{O}_{6}$ - тетрагональную структуру [13], $\mathrm{Cu}_{2} \mathrm{O}$ кристаллизуется в кубической решетке [14] и, наконец, соединение $\mathrm{La}_{2} \mathrm{CuO}_{4}$ кристаллизуется в решетке типа $\mathrm{K}_{2} \mathrm{NiF}_{4}$ (слабо искаженная ортогональная структура, причем для $x>0.1$ решетка $\mathrm{La}_{2-x} \mathrm{Sr}_{x} \mathrm{CuO}_{4}$ становится тетрагональной [13]).

Компоненты тензора решеточного ГЭП рассчитывались в рамках модели точечных зарядов по соотношениям

$$
\begin{gathered}
V_{\alpha \alpha}=\sum_{k} e_{k} \sum_{i} \frac{1}{r_{k i}^{3}}\left[\frac{3 \alpha_{k i}^{2}}{r_{k i}^{2}}-1\right]=\sum_{k} e_{k} G_{\alpha \alpha k}, \\
V_{\alpha \beta}=\sum_{k} e_{k} \sum_{i} \frac{3 \alpha_{k i} \beta_{k i}}{r_{k i}^{5}}=\sum_{k} e_{k} G_{\alpha \beta k},
\end{gathered}
$$

где $k-$ индекс суммирования по подрешеткам, $i$ индекс суммирования по узлам подрешетки, $\alpha, \beta-$ декартовы координаты, $e_{k}$ - эффективные заряды атомов $k$-подрешетки (в единицах заряда электрона $e$ ), $r_{k i}$ расстояние от $i$-иона $k$-подрешетки до узла, в котором рассчитывается ГЭП.

Решетка $\mathrm{Cu}_{2} \mathrm{O}$ представлялась в виде двух подрешеток и структурная формула записывалась в виде $\mathrm{Cu}_{4} \mathrm{O}_{2}$. Положения атомов в элементарной ячейке и параметры элементарной ячейки задавались согласно [14].

Элементарная ячейка $\mathrm{YBa}_{2} \mathrm{Cu}_{3} \mathrm{O}_{7}$, содержащая один узел иттрия, два эквивалентных узла бария, один цепочечный узел меди $\mathrm{Cu}(1)$, два эквивалентных планарных узла меди $\mathrm{Cu}(2)$, два эквивалентных апикальных узла кислорода $\mathrm{O}(1)$, четыре планарных узла кислорода (попарно эквивалентные узлы $\mathrm{O}(2)$ и $\mathrm{O}(3)$ ) и один цепочечный узел кислорода $\mathrm{O}(4)$, представлялась в виде: $\mathrm{YBa}_{2} \mathrm{Cu}(1) \mathrm{Cu}(2)_{2} \mathrm{O}(1)_{2} \mathrm{O}(2)_{2} \mathrm{O}(3)_{2} \mathrm{O}(4)$ (см. рис. 1, $\left.a\right)$, а индекс суммирования в (3) по подрешеткам принимал следующие значения:

$$
\begin{array}{ccccccccc}
k= & 1 & 2 & 3 & 4 & 5 & 6 & 7 & 8 \\
\text { атом } & \mathrm{Y} & \mathrm{Ba} & \mathrm{Cu}(1) & \mathrm{Cu}(2) & \mathrm{O}(1) & \mathrm{O}(2) & \mathrm{O}(3) & \mathrm{O}(4)
\end{array}
$$

Параметры элементарной ячейки для ромбоэдрической фазы и положение атомов в элементарной ячейке задавались согласно данным [13,15-18].

Структурная формула $\mathrm{YBa}_{2} \mathrm{Cu}_{3} \mathrm{O}_{6}$ записывалась в виде $\mathrm{YBa}_{2} \mathrm{Cu}(1) \mathrm{Cu}(2)_{2} \mathrm{O}(1)_{2} \mathrm{O}(2)_{2} \mathrm{O}(3)_{2}$, параметры элементарной ячейки для тетрагональной фазы задавались согласно [13].

В решетках твердых растворов $\mathrm{La}_{2-x} \mathrm{Sr}_{x} \mathrm{CuO}_{4}$ атомы лантана (стронция) и меди занимают единственные позиции, тогда как атомы кислорода занимают две неэквивалентные позиции, обозначаемые как $\mathrm{O}(1)$ (апикальный кислород) и $\mathrm{O}(2)$ (планарный кислород) 

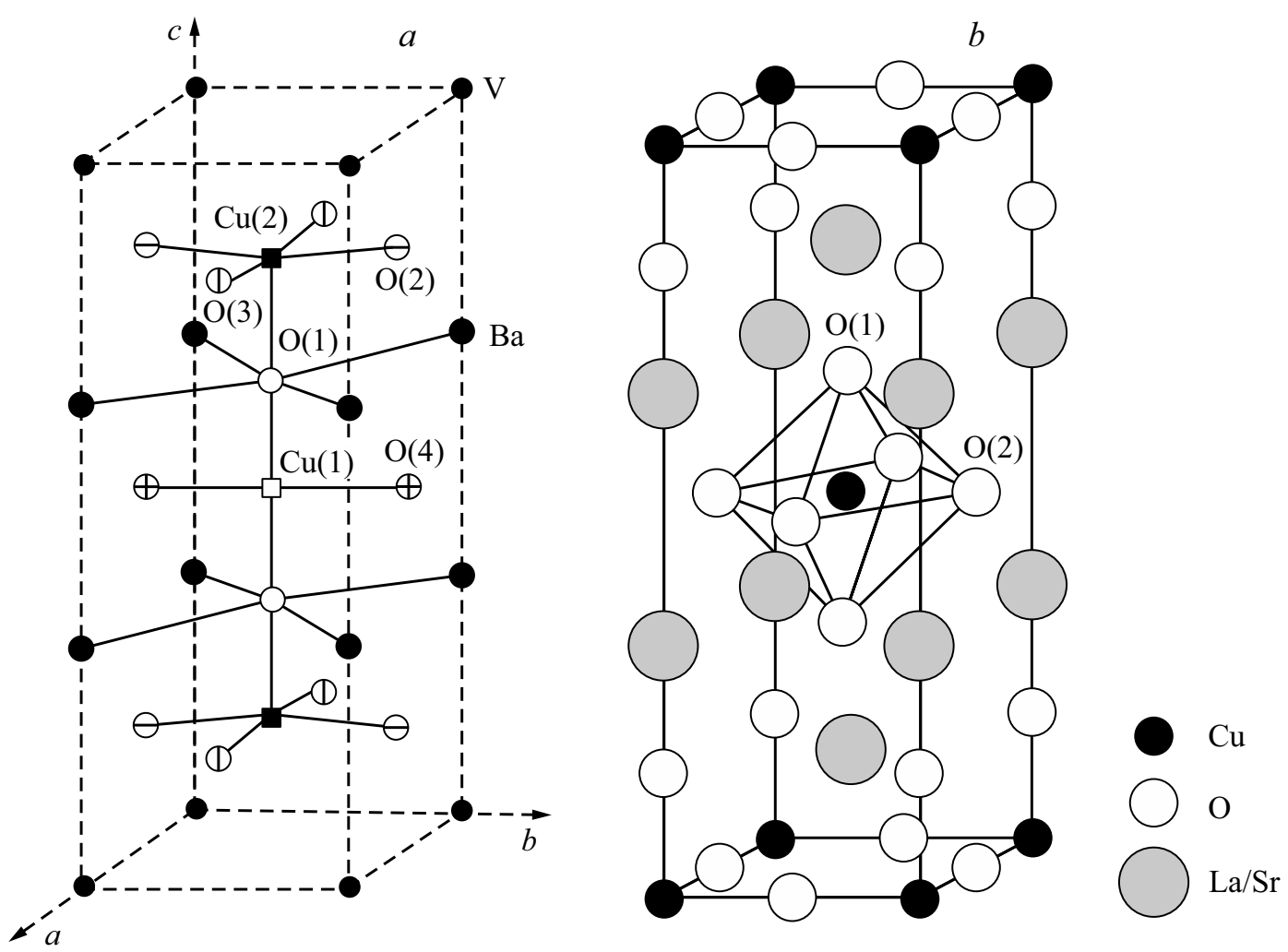

Рис. 1. Элементарные ячейки $\mathrm{YBa}_{2} \mathrm{Cu}_{3} \mathrm{O}_{7}(a)$ и $\mathrm{La}_{2-x} \mathrm{Sr}_{x} \mathrm{CuO}_{4}(b)$.

(рис. 1,b) [13]. Таким образом решетка $\mathrm{La}_{2-x} \mathrm{Sr}_{x} \mathrm{CuO}_{4}$ представлялась в виде четырех подрешеток и записывалась как $(\mathrm{La}, \mathrm{Sr}){ }_{2} \mathrm{CuO}(1)_{2} \mathrm{O}(2)_{2}$ (рис. 1, b). Зависимости параметров элементарной ячейки от $x$ взяты из [19], положение атомов в элементарной ячейке задавались согласно [13], а индекс суммирования в (3) по подрешеткам $k$ принимал следующие значения:

$$
\begin{array}{cccccc}
k= & 1 & 2 & 3 & 4 & 5 \\
\text { атом } & \mathrm{La} & \mathrm{Sr} & \mathrm{Cu} & \mathrm{O}(1) & \mathrm{O}(2)
\end{array}
$$

Решеточные суммы $G_{\alpha \alpha}$ и $G_{\beta \beta}$ подсчитывались на ЭВМ, суммирование проводилось внутри сфер радиуса $30 \AA$. Для решеток $\mathrm{YBa}_{2} \mathrm{Cu}_{3} \mathrm{O}_{7}, \mathrm{YBa}_{2} \mathrm{Cu}_{3} \mathrm{O}_{6}$ и $\mathrm{La}_{2-x} \mathrm{Sr}_{x} \mathrm{CuO}_{4}$ тензоры решеточных сумм $G_{k}$ от всех подрешеток (и, следовательно, суммарный тензор) были диагональными в кристаллографических осях. Тензор решеточного ГЭП для узлов меди решетки $\mathrm{Cu}_{2} \mathrm{O}$ в кристаллографических осях был недиагональным и была проведена его диагонализация. Полученные параметры тензоров ГЭП хорошо согласуются с литературными данными (см., например, [7]), особенностью наших расчетов является выделение вклада в тензор ГЭП от каждой подрешетки, что позволяет варьировать заряды ek при сопоставлении расчетных и измеренных параметров тензора ГЭП.

Под термином „эффективные заряды“ понимаются заряды, которые требуются для описания электрического поля ионов с помощью кулоновского потенциала. Эффективные заряды дают хорошее представление о валентных состояниях ионов в узлах решетки и о существенных отклонениях от стандартных валентных состояний.

2.2. Синтез образцов и измерение мессбауэровских спектров. Образцы $\mathrm{YBa}_{2} \mathrm{Cu}_{3} \mathrm{O}_{7}$ готовились по керамической технологии методом спекания спрессованных порошков оксидов меди, бария и иттрия, взятых в стехиометрических соотношениях $[13,17]$. Полученный материал был однофазным и имел орторомбическую структуру. Для температуры перехода в сверхпроводящее состояние получено значение $91 \mathrm{~K}$. Образцы $\mathrm{YBa}_{2} \mathrm{Cu}_{3} \mathrm{O}_{6}$ готовились отжигом $\mathrm{YBa}_{2} \mathrm{Cu}_{3} \mathrm{O}_{7}$ в вакууме при постоянной откачке. Полученные образцы были однофазными, имели тетрагональную структуру. Легирование $\mathrm{YBa}_{2} \mathrm{Cu}_{3} \mathrm{O}_{7}$ изотопами ${ }^{67} \mathrm{Cu}$ и ${ }^{67} \mathrm{Ga}$ проводилось в процессе диффузионного отжига в атмосфере кислорода при температурах $500-650^{\circ} \mathrm{C}$. Для контрольных образцов указанная термообработка не сопровождалась изменением структуры или $T_{c}$. Легирование $\mathrm{YBa}_{2} \mathrm{Cu}_{3} \mathrm{O}_{7}$ и $\mathrm{YBa}_{2} \mathrm{Cu}_{3} \mathrm{O}_{6}$ изотопами ${ }^{67} \mathrm{Cu}$ и ${ }^{67} \mathrm{Ga}$ проводилось в процессе диффузионного отжига в вакууме при температуре $650^{\circ} \mathrm{C}$.

Синтез образцов $\mathrm{La}_{2-x} \mathrm{Sr}_{x} \mathrm{CuO}_{4}(x=0.1-1.0)$ проводили по керамической технологии путем спекания смеси оксидов меди, лантана и карбоната стронция [19]. Полученные образцы с $x \leq 0.3$ были однофазными, имели структуру типа $\mathrm{K}_{2} \mathrm{NiF}_{4}$ с температурами перехода в сверхпроводящее состояние $T_{c}=25,37,32$ и $<4.2 \mathrm{~K}$ 
соответственно для $x=0.1,0.15,0.2$ и $0.3-1.0$ (и это согласуется с литературными данными [19]). Образцы с $x \geq 0.4$ содержали небольшую $(<5 \%)$ фракцию второй фазы. Мессбауэровские источники $\mathrm{La}_{2-x} \mathrm{Sr}_{x} \mathrm{CuO}_{4}:{ }^{57} \mathrm{Co}$ и $\mathrm{La}_{2-x} \mathrm{Sr}_{x} \mathrm{CuO}_{4}::^{155} \mathrm{Eu}$ готовились путем добавления нитратов кобальта или европия, меченых радиоактивными изотопами ${ }^{57} \mathrm{Co}$ или ${ }^{155} \mathrm{Eu}$, в исходную шихту. Мессбауэровские источники $\mathrm{La}_{2-x} \mathrm{Sr}_{x} \mathrm{CuO}_{4}:{ }^{67} \mathrm{Cu}$ и $\mathrm{La}_{2-x} \mathrm{Sr}_{x} \mathrm{CuO}_{4}:{ }^{.67} \mathrm{Ga}$ готовились методом диффузии соответствующих короткоживущих изотопов в готовую керамику при температурах $500-650^{\circ} \mathrm{C}$ в течение $2 \mathrm{~h}$ в атмосфере кислорода. Для нелегированных образцов $\mathrm{La}_{2-x} \mathrm{Sr}_{x} \mathrm{CuO}_{4}$ отжиг в аналогичных условиях не привел к изменению величин $T_{c}$.

Закись меди $\mathrm{Cu}_{2} \mathrm{O}$ была получена прокалкой $\mathrm{CuO}$ в вакууме при постоянной откачке [14]. Окись меди $\mathrm{CuO}$ получали осаждением гидроксида меди из водного раствора медного купороса и его прокаливанием на воздухе. Радиоактивный изотоп ${ }^{67} \mathrm{Cu}$ вводили в раствор медного купороса в виде слабокислого раствора. Однофазность образцов контролировалась рентгенофазовым анализом.

Мессбауэровские спектры снимались при $80 \mathrm{~K}$ $\left({ }^{57} \mathrm{Co},{ }^{155} \mathrm{Eu}\right)$ и $4.2 \mathrm{~K}\left({ }^{67} \mathrm{Cu},{ }^{67} \mathrm{Ga}\right)$ с поглотителями $\mathrm{K}_{4}^{57} \mathrm{Fe}(\mathrm{CN})_{6.3} \mathrm{H}_{2} \mathrm{O},{ }^{155} \mathrm{GdPd}_{3}$ и ${ }^{67} \mathrm{ZnS}$.

\section{3. Экспериментальные результаты и их обсуждение}

3.1. Данные эмС. Типичные эмиссионные мессбауэровские спектры приведены на рис. 2, $a$ результаты их обработки сведены на рис. 3 и 4, а также в табл. 1 вместе с экспериментальными значениями $C_{\text {exp }}, \eta_{\text {exp }}$ и ориентациями $z$-осей тензоров ГЭП для узлов бария $[6,20]$ и кислорода [2,3], определенными методами ЯМР/ЯКР.

В эмиссионном мессбауэровском спектре ${ }^{67} \mathrm{Cu}\left({ }^{67} \mathrm{Zn}\right)$ сверхпроводящей керамики $\mathrm{YBa}_{2} \mathrm{Cu}_{3} \mathrm{O}_{7}$ наблюдаются два квадрупольных триплета с различными значениями $e Q U_{z z}$ и отношением интенсивностей $\sim 1: 2$ (рис. 2). Менее интенсивный спектр следует отнести к центрам ${ }^{67} \mathrm{Zn}^{2+}$ в узлах $\mathrm{Cu}(1)$, а более интенсивный - к центрам ${ }^{67} \mathrm{Zn}^{2+}$ в узлах $\mathrm{Cu}(2)$. Для керамики $\mathrm{YBa}_{2} \mathrm{Cu}_{3} \mathrm{O}_{6}$ мессбауэровский спектр ${ }^{67} \mathrm{Cu}\left({ }^{67} \mathrm{Zn}\right)$ представляет собой квадрупольный триплет, который следует отнести к центрам ${ }^{67} \mathrm{Zn}^{2+}$ в узлах $\mathrm{Cu}(1)$, а отсутствие второго триплета объясняется антиферромагнитным упорядочением подрешетки $\mathrm{Cu}(2)$. В закиси меди $\mathrm{Cu}_{2} \mathrm{O}$ и твердых растворах $\mathrm{La}_{2-x} \mathrm{Sr}_{x} \mathrm{CuO}_{4}$ атомы меди занимают единственную позицию, локальная симметрия которой ниже кубической, и, как результат, мессбауэровские спектры образцов $\mathrm{Cu}_{2} \mathrm{O}::^{57} \mathrm{Cu}$ и $\mathrm{La}_{2-x} \mathrm{Sr}_{x} \mathrm{CuO}_{4}:{ }^{67} \mathrm{Cu}$ представляют собой квадрупольные триплеты, отвечающие центрам $\mathrm{Zn}^{2+}$ в узлах меди (рис. 2).

Атомы иттрия и лантана занимают единственную кристаллографическую позицию в решетках $\mathrm{YBa}_{2} \mathrm{Cu}_{3} \mathrm{O}_{7}$ и $\mathrm{La}_{2-x} \mathrm{Sr}_{x} \mathrm{CuO}_{4}$ и поэтому эмиссионные мессбауэровские

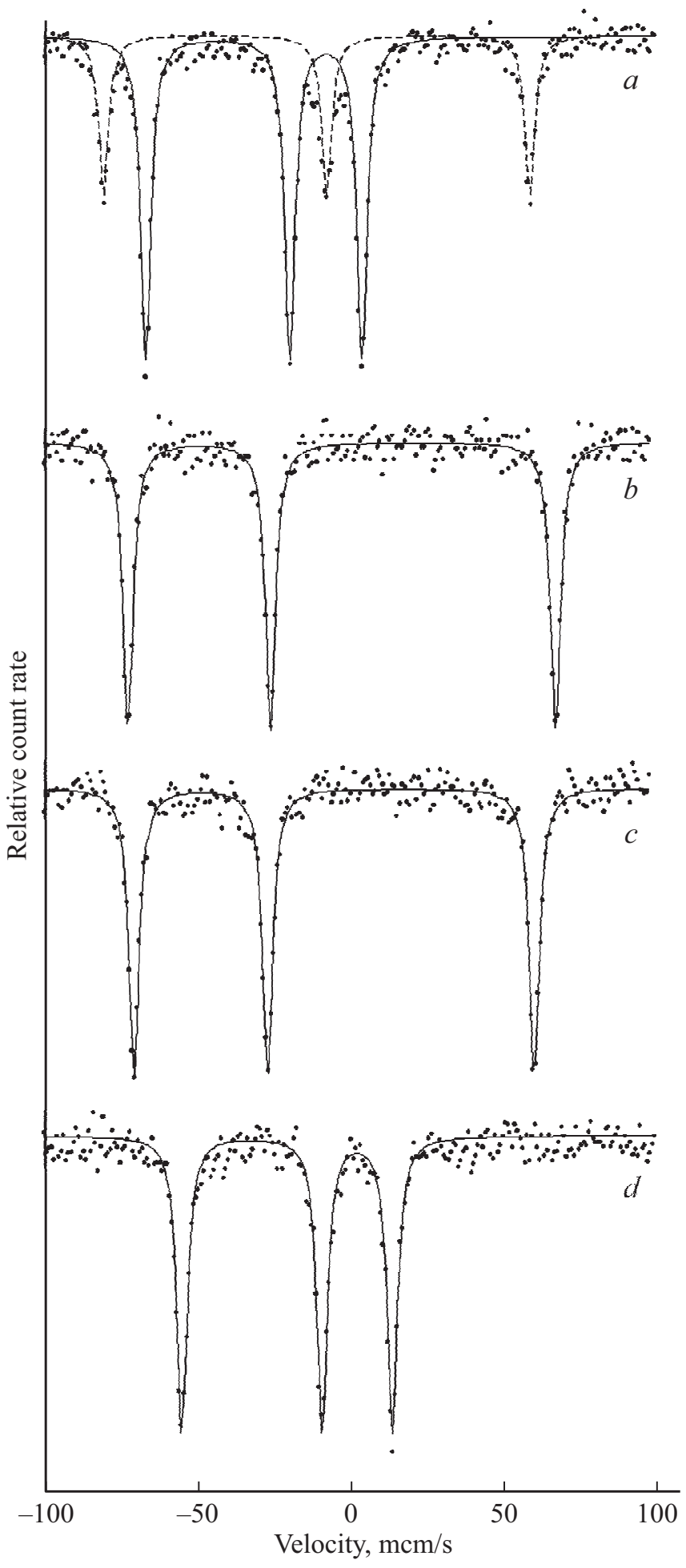

Рис. 2. Эмиссионные мессбауэровские спектры ${ }^{67} \mathrm{Cu}\left({ }^{67} \mathrm{Zn}\right)$ соединений $\mathrm{YBa}_{2} \mathrm{Cu}_{3} \mathrm{O}_{7}(a), \mathrm{YBa}_{2} \mathrm{Cu}_{3} \mathrm{O}_{6}(b), \mathrm{Cu}_{2} \mathrm{O}$ (c) и $\mathrm{La}_{1.85} \mathrm{Sr}_{0.15} \mathrm{CuO}_{4}(d)$ при $4.2 \mathrm{~K}$.

спектры ${ }^{67} \mathrm{Ga}\left({ }^{67} \mathrm{Zn}\right)$ отвечает одному состоянию центра ${ }^{67} \mathrm{Zn}^{2+}$ в узлах $\mathrm{Y}$ и La. Некубическая симметрия локального окружения атомов Y и La объясняет появление спектра в виде квадрупольного триплета. 
Таблица 1. Экспериментальные параметры ЯКВ в узлах решеток $\mathrm{YBa}_{2} \mathrm{Cu}_{3} \mathrm{O}_{7}, \mathrm{YBa}_{2} \mathrm{Cu}_{3} \mathrm{O}_{6}$ и $\mathrm{Cu}_{2} \mathrm{O}$

\begin{tabular}{|c|c|c|c|c|c|c|c|}
\hline Соединение & Узел & Зонд & Метод & $C_{\text {exp }}, \mathrm{MHz}$ & $\eta_{\exp }$ & $\begin{array}{c}z \text {-ось } \\
\text { тензора ГЭП }\end{array}$ & Ссылка \\
\hline $\mathrm{YBa}_{2} \mathrm{Cu}_{3} \mathrm{O}_{7}$ & $\begin{array}{c}\mathrm{Y} \\
\mathrm{Ba} \\
\mathrm{Cu}(1) \\
\mathrm{Cu}(2) \\
\mathrm{O}(1) \\
\mathrm{O}(2) \\
\mathrm{O}(3) \\
\mathrm{O}(4)\end{array}$ & $\begin{array}{l}{ }^{67} \mathrm{Zn} \\
{ }^{137} \mathrm{Ba} \\
{ }^{67} \mathrm{Zn} \\
{ }^{67} \mathrm{Zn} \\
{ }^{17} \mathrm{O} \\
{ }^{17} \mathrm{O} \\
{ }^{17} \mathrm{O} \\
{ }^{17} \mathrm{O}\end{array}$ & $\begin{array}{c}\text { ЭМС }{ }^{67} \mathrm{Ga}\left({ }^{67} \mathrm{Zn}\right) \\
\text { ЯМР/ЯКР }{ }^{137} \mathrm{Ba} \\
\text { ЭМС }{ }^{67} \mathrm{Cu}\left({ }^{67} \mathrm{Zn}\right) \\
\text { ЭМС }{ }^{67} \mathrm{Cu}\left({ }^{67} \mathrm{Zn}\right) \\
\text { ЯMP }{ }^{17} \mathrm{O} \\
\text { ЯMP }{ }^{17} \mathrm{O} \\
\text { ЯMP }{ }^{17} \mathrm{O} \\
\text { ЯMР }{ }^{17} \mathrm{O}\end{array}$ & $\begin{array}{c}-2.2(3) \\
|56.4(1)| \\
+20.1(3) \\
+11.8(3) \\
|7.3(1)| \\
|6.4(1)| \\
|6.6(1)| \\
|10.9(1)|\end{array}$ & $\begin{array}{c}0.8(1) \\
0.94(2) \\
0.95(3) \\
\leq 0.2 \\
0.32(2) \\
0.24(2) \\
0.21(2) \\
0.41(2)\end{array}$ & $\begin{array}{l}c \\
b \\
a \\
b\end{array}$ & $\begin{array}{l}{[*]} \\
{[6]} \\
{[*]} \\
{[*]} \\
{[2]} \\
{[2]} \\
{[2]} \\
{[2]}\end{array}$ \\
\hline $\mathrm{La}_{1.85} \mathrm{Sr}_{0.15} \mathrm{CuO}_{4}$ & $\begin{array}{l}\mathrm{La}, \mathrm{Sr} \\
\mathrm{Cu} \\
\mathrm{O}(1) \\
\mathrm{O}(2)\end{array}$ & $\begin{array}{l}{ }^{67} \mathrm{Zn} \\
{ }^{67} \mathrm{Zn} \\
{ }^{17} \mathrm{O} \\
{ }^{17} \mathrm{O}\end{array}$ & $\begin{array}{c}\text { ЭМС }{ }^{67} \mathrm{Cu}\left({ }^{67} \mathrm{Zn}\right) \\
Э \mathrm{MC}{ }^{67} \mathrm{Cu}\left({ }^{67} \mathrm{Zn}\right) \\
\text { ЯMP }{ }^{17} \mathrm{O} \\
\text { ЯMP }{ }^{17} \mathrm{O}\end{array}$ & $\begin{array}{c}-2.7(2) \\
11.4(5) \\
|1.33(5)| \\
|4.6(1)|\end{array}$ & $\begin{array}{c}\leq 0.2 \\
\leq 0.2 \\
0.0 \\
0.36(2)\end{array}$ & & $\begin{array}{l}{[*]} \\
{[*]} \\
{[3]} \\
{[3]}\end{array}$ \\
\hline $\mathrm{YBa}_{2} \mathrm{Cu}_{3} \mathrm{O}_{6}$ & $\begin{array}{c}\mathrm{Cu}(1) \\
\mathrm{Ba}\end{array}$ & $\begin{array}{l}{ }^{67} \mathrm{Zn} \\
{ }^{137} \mathrm{Ba}\end{array}$ & $\begin{array}{c}\text { ЭМС }{ }^{67} \mathrm{Cu}\left({ }^{67} \mathrm{Zn}\right) \\
\text { ЯМР/ЯКР }{ }^{137} \mathrm{Ba}\end{array}$ & $\begin{array}{l}-23.5(3) \\
|80.3(1)|\end{array}$ & $\begin{array}{c}\leq 0.2 \\
0\end{array}$ & & $\begin{array}{c}{[*]} \\
{[20]}\end{array}$ \\
\hline $\mathrm{Cu}_{2} \mathrm{O}$ & $\mathrm{Cu}$ & ${ }^{67} \mathrm{Zn}$ & ЭМС ${ }^{67} \mathrm{Cu}\left({ }^{67} \mathrm{Zn}\right)$ & $-22.0(3)$ & $\leq 0.2$ & & {$[*]$} \\
\hline
\end{tabular}

Примечание. * - результаты настоящей работы.

Мессбауэровские спектры образцов $\mathrm{La}_{2-x} \mathrm{Sr}_{x} \mathrm{CuO}_{4}::^{57} \mathrm{Co}$ и $\mathrm{La}_{2-x} \mathrm{~S}_{r x} \mathrm{CuO}_{4}:{ }^{155} \mathrm{Eu}$ представляют собой квадрупольные дублеты, изомерный сдвиг которых отвечает трехвалентным атомам железа и европия и их следует отнести к центрам ${ }^{57 m} \mathrm{Fe}^{3+}$ в узлах меди и ${ }^{155} \mathrm{Ga}$ в узлах лантана.

\section{2. Определение коэффициента $\alpha_{\mathrm{Zn}}=e Q_{\mathrm{Zn}}$} $\times\left(1-\gamma_{\mathrm{Zn}}\right)$ для зонда ${ }^{67} \mathrm{Zn}^{2+}$. Для определения эффективных зарядов атомов в решетках $\mathrm{YBa}_{2} \mathrm{Cu}_{3} \mathrm{O}_{7}$

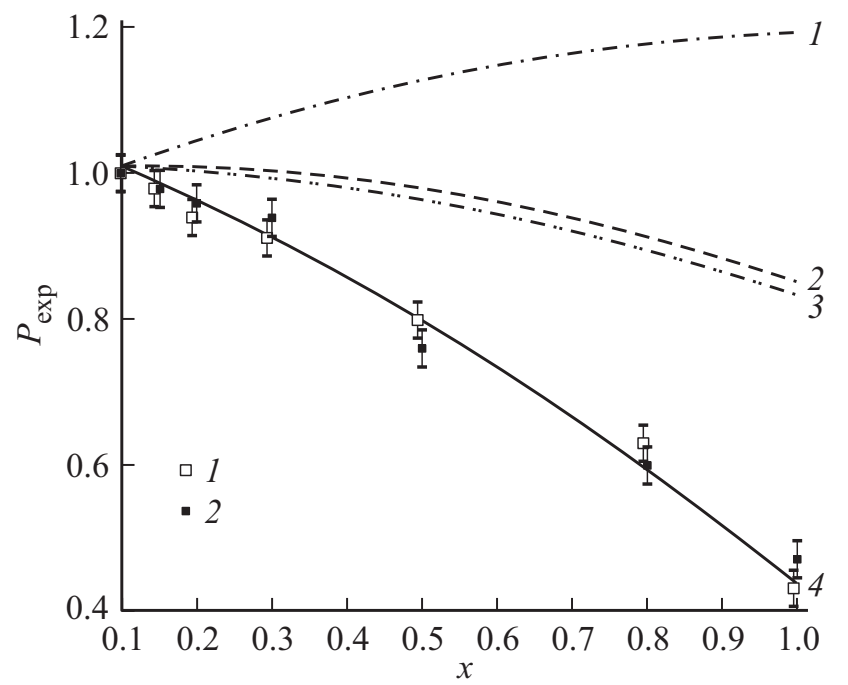

Рис. 3. Зависимости $P_{\exp }$ от $x$ для $\mathrm{La}_{2-x} \mathrm{Sr}_{x} \mathrm{CuO}_{4}$ для узлов меди: (1) дырка находится в подрешетке меди; (2) дырка находится в подрешетке $\mathrm{O}(1) ;(3)$ дырка находится в подрешетке $\mathrm{O}(2) ;(4)$ дырка распределена между подрешетками $\mathrm{O}(1)$ и $\mathrm{O}(2)$; светлые и залитые квадраты - экспериментальные данные ЭМС на изотопах ${ }^{57} \mathrm{Co}\left({ }^{57 m} \mathrm{Fe}\right)(1)$ и ${ }^{67} \mathrm{Cu}\left({ }^{67} \mathrm{Zn}\right)(2)$. и $\mathrm{La}_{2-x} \mathrm{Sr}_{x} \mathrm{CuO}_{4}$ нами были определены величины $\alpha_{\mathrm{Zn}}$ для зонда ${ }^{67} \mathrm{Zn}^{2+}$ путем сравнения экспериментальных величин $C_{\text {exp }}$ и расчетных величин $V_{z z}$ для узлов меди в кристаллических решетках $\mathrm{Cu}_{2} \mathrm{O}$ и $\mathrm{YBa}_{2} \mathrm{Cu}_{3} \mathrm{O}_{6}$.

Если принять модели распределения зарядов по узлам указанных решеток соответствующих стандартным валентным состояниям всех атомов (т.е. $\mathrm{Cu}_{2}^{+} \mathrm{O}^{2-}$ и $\left.\mathrm{Y}^{3+} \mathrm{Ba}_{2}^{2+} \mathrm{Cu}(1)^{+} \mathrm{Cu}(2)_{2}^{2+} \mathrm{O}_{6}^{2-}\right)$, то расчет узлов меди в $\mathrm{Cu}_{2} \mathrm{O}$ дает $V_{z z}=-1.093 \mathrm{e} / \AA^{3}$ и для уз-

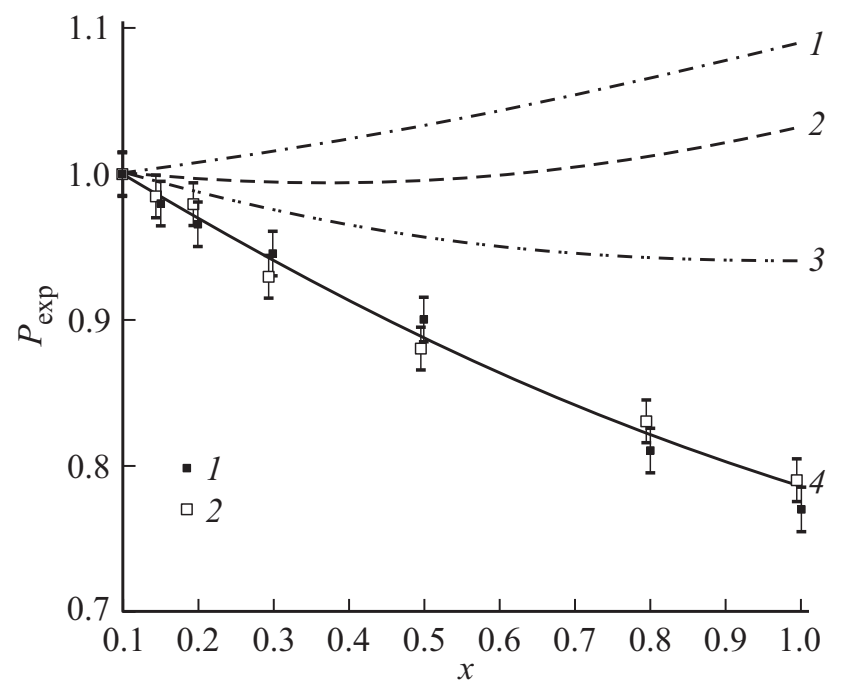

Рис. 4. Зависимости $P_{\exp }$ от $x$ для $\mathrm{La}_{2-x} \mathrm{Sr}_{x} \mathrm{CuO}_{4}$ для узлов лантана: 1 - дырка локализована в позициях $\mathrm{Cu} ; 2$ - дырка локализована в позициях $\mathrm{O}(1) ; 3$ - дырка локализована в позициях $\mathrm{O}(2) ; 4$ - дырка локализована в позициях $\mathrm{O}(1)$ и $\mathrm{O}(2)$; светлые и залитые квадраты - экспериментальные данные на изотопах ${ }^{67} \mathrm{Ga}\left({ }^{67} \mathrm{Zn}\right)$ (1) и ${ }^{155} \mathrm{Eu}\left({ }^{155} \mathrm{Gd}\right)$ (2). 
лов $\mathrm{Cu}(1)$ в $\mathrm{YBa}_{2} \mathrm{Cu}_{3} \mathrm{O}_{6} \quad V_{z z}=-1.253 \mathrm{e} / \AA^{3}$. Это приводит к величинам $\alpha_{Z n}$, равным 20.1(3) MHz $\cdot \AA^{3} / \mathrm{e}$ и $18.8(3) \mathrm{MHz} \cdot \AA^{3} /$ е для центров ${ }^{67} \mathrm{Zn}^{2+}$ в узлах меди решетки $\mathrm{Cu}_{2} \mathrm{O}$ и в узлах $\mathrm{Cu}(1)$ решетки $\mathrm{YBa}_{2} \mathrm{Cu}_{3} \mathrm{O}_{6}$ соответственно. Таким образом, коэффициенты $\alpha_{\mathrm{Zn}}$ несколько различаются ( $\pm 3.3 \%$ относительно среднего значения) при замещении зондом ${ }^{67} \mathrm{Zn}^{2+}$ одновалентной меди в различных кристаллических решетках. Для дальнейших расчетов мы приняли значение $\alpha_{\mathrm{Zn}}=20.1(3) \mathrm{MHz} \cdot \AA^{3} / \mathrm{e}$, т. к. оно получено в предположении только о зарядах кислорода в $\mathrm{Cu}_{2} \mathrm{O}$ (вклад в тензор ГЭП в узлах меди от подрешетки меди отсутствует), а для $\mathrm{YBa}_{2} \mathrm{Cu}_{3} \mathrm{O}_{6}$ требуется задать заряды еще четырех катионов.

3.3. Определение эффективных зарядов атомов решетки $\mathrm{YBa}_{2} \mathrm{Cu}_{3} \mathrm{O}_{7}$. Для определения эффективных зарядов восьми атомов решетки $\mathrm{YBa}_{2} \mathrm{Cu}_{3} \mathrm{O}_{7}$ использовались данные ЭМС на изотопах ${ }^{67} \mathrm{Cu}\left({ }^{67} \mathrm{Zn}\right)$ и ${ }^{67} \mathrm{Ga}\left({ }^{67} \mathrm{Zn}\right)$, а также данные ЯМР на изотопе ${ }^{17} \mathrm{O}$ [2] (поскольку в [7] было показано, что в узлах $\mathrm{O}(1)$ и $\mathrm{O}(2)$ зонды кислорода можно считать кристаллическими).

Для определения восьми эффективных зарядов предлагается составить систему восьми уравнений [21]:

$$
\begin{gathered}
e_{1}+2 e_{2}+e_{3}+2 e_{4}+2 e_{5}+2 e_{6}+2 e_{7}+e_{8}=0 \\
\alpha_{\mathrm{Zn}} \sum_{k=1}^{k=8} e_{k} G_{z z k 1}=C_{1},
\end{gathered}
$$

$\sum_{k=1}^{k=8} y_{k}\left[G_{z z k 3}-P_{34} G_{z z k 4}\right]=0, \quad$ где $P_{34}=C_{3} / C_{4}$,

$\sum_{k=1}^{k=8} e_{k}\left[G_{z z k 5}-P_{56} G_{z z k 6}\right]=0, \quad$ где $P_{56}=C_{5} / C_{6}$,

$\sum_{k=1}^{k=8} e_{k}\left[G_{x x k l}-G_{y y k l}-\eta_{l} G_{z z k l}\right]=0, \quad$ где $l=3,4,5,6$.

Неопределенность знака постоянной квадрупольного взаимодействия для зонда ${ }^{17} \mathrm{O}$ требует подстановки в уравнение (7) величин $P_{56}$ как с положительным, так и с отрицательным знаком. Как не имеющие физического смысла были отброшены решения с отрицательными зарядами катионов или положительными зарядами анионов.

Все полученные эффективные заряды атомов с использованием данных табл. 1 и $\alpha_{\mathrm{Zn}}=20.1 \mathrm{MHz} \cdot \mathrm{AA}^{3} / \mathrm{e}$ отвечают пониженному заряду атомов цепочечного кислорода $\mathrm{O}(4)$ и заметному отклонения от стандартного значения заряда для атомов планарного кислорода $\mathrm{O}(3)$, что может быть интерпретировано как существование дырки в энергетической зоне, образованной преимущественно электронными состояниями $\mathrm{O}(4)$ и $\mathrm{O}(3)$. В качестве примера приведем распределение зарядов
Таблица 2. Компоненты тензоров решеточных ГЭП для узлов кристалла $\mathrm{YBa}_{2} \mathrm{Cu}_{3} \mathrm{O}_{7}$ (модель $A$ )

\begin{tabular}{c|c|c|c|c}
\hline Узел & $V_{a a}, \mathrm{e} / \AA^{3}$ & $V_{b b}, \mathrm{e} / \AA^{3}$ & $V_{c c}, \mathrm{e} / \AA^{3}$ & $\eta_{\text {lat }}$ \\
\hline $\mathrm{Y}$ & 0.006 & 0.107 & -0.113 & 0.89 \\
$\mathrm{Ba}$ & -0.118 & -0.003 & 0.121 & 0.94 \\
$\mathrm{Cu}(1)$ & 0.982 & -0.036 & -0.946 & 0.97 \\
$\mathrm{Cu}(2)$ & -0.263 & -0.324 & 0.587 & 0.10 \\
$\mathrm{O}(1)$ & -0.158 & -0.331 & 0.489 & 0.35 \\
$\mathrm{O}(2)$ & -0.153 & 0.385 & -0.232 & 0.21 \\
$\mathrm{O}(3)$ & 0.439 & -0.206 & -0.233 & 0.06 \\
$\mathrm{O}(4)$ & -0.086 & 0.575 & -0.489 & 0.70
\end{tabular}

атомов, полученное при использовании экспериментальных величин из табл. 1 и структурных данных [13,17]:

$$
\mathrm{Y}^{3+} \mathrm{Ba}_{2}^{2+} \mathrm{Cu}(1)^{2+} \mathrm{Cu}(2)_{2}^{2+} \mathrm{O}(1)_{2}^{2-} \mathrm{O}(2)_{2}^{2-} \mathrm{O}(3)_{2}^{1.85-} \mathrm{O}^{1.3-} \text {. }
$$

Для всех полученных моделей зарядового распределения расчетные параметры тензоров решеточного ГЭП оказываются близкими для структурных данных [13] и [17], и для модели $A$ эти величины сведены в табл. 2.

Отметим, что величины эффективных зарядов зависят от температуры определения структурных параметров, а также различаются при использовании структурных данных разных авторов [13,15-18]. Однако отклонения зарядов всех атомов, кроме $\mathrm{O}(4)$ и $\mathrm{O}(3)$, от стандартных степеней окисления относительно малы, и, варьируя значения экспериментальных величин из табл. 1 в пределах их погрешностей, можно получить заряды, достаточно близкие к стандартным степеням окисления.

Полученные модели зарядового распределения можно использовать для интерпретации данных ЯМР/ЯКР на изотопе ${ }^{137} \mathrm{Ba}$, для которого были найдены значения $C_{\exp }, \eta_{\exp }$ в $\mathrm{YBa}_{2} \mathrm{Cu}_{3} \mathrm{O}_{6}$ [20] и в $\mathrm{YBa}_{2} \mathrm{Cu}_{3} \mathrm{O}_{7}$ [6] (см. табл. 1). В приближении точечных зарядов параметры тензора решеточного ГЭП в узлах Ва для модели $\mathrm{Y}^{3+} \mathrm{Ba}_{2}^{2+} \mathrm{Cu}(1)^{+} \mathrm{Cu}(2)_{2}^{2+} \mathrm{O}_{6}^{2-}$ было получено $V_{z z}=0.1572 \mathrm{e} / \AA^{3}, \eta=0$, а также были определены значения $V_{z z}$ и $\eta$ для узлов бария в решетке $\mathrm{YBa}_{2} \mathrm{Cu}_{3} \mathrm{O}_{7}$ (см. табл. 2). В итоге, значения коэффициента $\alpha_{\mathrm{Ba}}=e Q(1-\gamma)$ для зонда ${ }^{137} \mathrm{Ba}^{2+}$ оказались

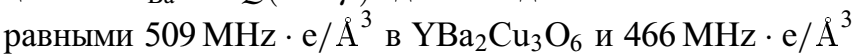
в $\mathrm{YBa}_{2} \mathrm{Cu}_{3} \mathrm{O}_{7}$. Эти значения различаются на $\pm 4.4 \%$ относительно среднего значения.

Для сопоставления расчетных и экспериментальных результатов в узлах Ү можно воспользоваться данными мессбауэровской спектроскопии на изотопе ${ }^{155} \mathrm{Gd}$ в соединении $\mathrm{GdBa}_{2} \mathrm{Cu}_{3} \mathrm{O}_{7}$ (в этом соединении узлы $\mathrm{Gd}$ являются аналогами узлов $\mathrm{Y}$ в $\left.\mathrm{YBa}_{2} \mathrm{Cu}_{3} \mathrm{O}_{7}\right)$ [22]. Для кристаллического зонда ${ }^{155} \mathrm{Gd}^{3+}$ было получено: $z \| c$ и $U_{z z}<0$, что находится в согласии с результатами расчетов методом точечных зарядов. Расчетные (методом точечных зарядов) и экспериментальные параметры 
асимметрии тензора ГЭП также находятся в удовлетворительном согласии.

\section{4. Эффективные заряды атомов решеток твер-} дых растворов $\mathrm{La}_{2-x} \mathrm{Sr}_{x} \mathrm{CuO}_{4}$. Для определения эффективных зарядов атомов кристаллических решеток $\mathrm{La}_{2-x} \mathrm{Sr}_{x} \mathrm{CuO}_{4}$ необходимо составить систему четырех уравнений. Использование данных ЭМС на изотопах ${ }^{67} \mathrm{Cu}\left({ }^{67} \mathrm{Zn}\right)$ и ${ }^{67} \mathrm{Ga}\left({ }^{67} \mathrm{Zn}\right)$ позволяет сформировать три уравнения:

$$
\begin{gathered}
2 e_{1}+e_{2}+2 e_{3}+2 e_{4}=0, \\
\alpha_{\mathrm{Zn}} \sum_{k=1}^{k=4} e_{k} \mathrm{G}_{z z k 1}=C_{1}, \\
\alpha_{\mathrm{Zn}} \sum_{k=1}^{k=4} e_{k} \mathrm{G}_{z z k 2}=0 .
\end{gathered}
$$

Для сформирования четвертого уравнения мы воспользовались данными ЯМР на изотопе ${ }^{17} \mathrm{O}$ для состава $\mathrm{La}_{1.85} \mathrm{Sr}_{0.15} \mathrm{CuO}_{4}$ [3] (см. табл. 1). Естественно, возникает вопрос, какой кислород (апикальный или планарный) рассматривать в качестве кристаллического зонда. В связи с этим отметим, что для решетки $\mathrm{La}_{1.85} \mathrm{Sr}_{0.15} \mathrm{CuO}_{4}$ главные оси тензоров решеточного ГЭП для всех узлов совпадают с кристаллографической осью $c$, и все тензоры аксиально-симметричны. С другой стороны, согласно данным ЯМР на изотопе ${ }^{17} \mathrm{O}$, параметр асимметрии тензора ГЭП для узлов планарного кислорода отличен от нуля (см. табл. 1), что, очевидно, свидетельствует о валентном вкладе в суммарный ГЭП. Иными словами, кристаллическим зондом может быть центр апикального кислорода и, следовательно, четвертым уравнением может служить уравнение для рассчитанной главной компоненты тензора ГЭП и экспериментальной постоянной квадрупольного взаимодействия зонда ${ }^{17} \mathrm{O}$ в узлах $\mathrm{O}(1)$ решетки $\mathrm{La}_{1.85} \mathrm{Sr}_{0.15} \mathrm{CuO}_{4}$

$$
\alpha_{\mathrm{O}}=\sum_{k=1}^{k=4} e_{k} G_{z z k 3}=0,
$$

где величина $\alpha_{\mathrm{O}}=14.9 \mathrm{MHz} \cdot \mathrm{AA}^{3} /$ е была определена путем сравнения экспериментального значения постоянной квадрупольного взаимодействия в узлах $\mathrm{O}(1)$ решетки $\mathrm{YBa}_{2} \mathrm{Cu}_{3} \mathrm{O}_{7}$ (данные ЯМР на изотопе ${ }^{17} \mathrm{O}$ [2], см. табл. 1) и расчетного значения $V_{z z 5}$ для модели $(A)$ (см. табл. 2).

Эффективные заряды атомов решетки $\mathrm{La}_{1.85} \mathrm{Sr}_{0.15} \mathrm{CuO}_{4}$, полученные с использованием данных табл. 1 и величин $\alpha_{\mathrm{Zn}}=20.1 \mathrm{MHz} \cdot \mathrm{AA}^{3} / \mathrm{e}$ и $\alpha_{\mathrm{O}}=14.9 \mathrm{MHz} \cdot \mathrm{AA}^{3} / \mathrm{e}$, отвечают пониженному заряду атомов планарного кислорода, что является следствием локализации дырки в энергетической зоне, образованной преимущественно электронными состояниями О(2). Отклонения зарядов всех атомов, кроме планарного кислорода, от стандартных степеней окисления относительно малы, и, варьируя значения экспериментальных величин из табл. 1 в пределах их погрешностей, можно получить заряды, отвечающие стандартным степеням окисления

$$
\left(\mathrm{La}_{1.85} \mathrm{Sr}_{0.15}\right)^{2.925+} \mathrm{Cu}^{2+} \mathrm{O}(1)_{2}^{2-}(2)_{2}^{1.925-}
$$

Подтверждение предложенной модели пространственного распределения электронных дефектов в решетке $\mathrm{La}_{1.85} \mathrm{Sr}_{0.15} \mathrm{CuO}_{4}$ может быть получено в результате совместного анализа результатов расчетных зависимостей $P(x)$ (здесь $P=\left[V_{z z}\right]_{x} /\left[V_{z z}\right]_{x}=0.1$ ) и экспериментальных зависимостей $P_{\exp }(x)$ (здесь $\left.P_{\exp }=\left[e Q U_{z z}\right]_{x} /\left[e Q U_{z z}\right]_{x=0}\right)$ в катионных узлах решеток $\mathrm{La}_{2-x} \mathrm{Sr}_{x} \mathrm{CuO}_{4}(x=0.1-1.0)$, полученных с использованием ЭМС на изотопах ${ }^{5} 7 \mathrm{Co}\left({ }^{57 m} \mathrm{Fe}\right),{ }^{67} \mathrm{Cu}\left({ }^{67} \mathrm{Zn}\right)$, ${ }^{67} \mathrm{Ga}\left({ }^{67} \mathrm{Zn}\right)$ и ${ }^{155} \mathrm{Eu}\left({ }^{155} \mathrm{Gd}\right)$. Зависимости $\mathrm{P}(\mathrm{x})$ для узлов меди и лантана приведены на рис. 3 и 4, причем расчет проведен для четырех моделей: (1) дырка находится в подрешетке меди; (2) дырка находится в подрешетке апикального кислорода; (3) дырка находится в подрешетке планарного кислорода; (4) дырка распределена между подрешетками апикального и планарного кислорода. К данным, соответствующим $x \geq 0.4$, следует относиться с некоторой осторожностью, поскольку для этих составов наблюдалась примесь второй фазы [12]. Однако в измеренных нами мессбауэровских спектрах дополнительных линий не было обнаружено.

Зависимости $P(x)$ для всех узлов описываются квадратичными полиномами, что отличается от данных авторов [12], которые в области составов от $x=0$ до $x=0.3$ получили линейные зависимости. Как видно из рис. 3 и 4, уменьшение величины $e Q U_{z z}$ в узлах меди и лантана с ростом $x$ для центров ${ }^{67} \mathrm{Zn}^{2+},{ }^{57 m} \mathrm{Fe}^{3+}$ и ${ }^{155} \mathrm{Gd}^{3+}$ может быть количественно объяснено, если дырка локализуется преимущественно в позициях планарного кислорода.

\section{4. Заключение}

Эффективные заряды всех атомов решеток сверхпроводящих керамик $\mathrm{YBa}_{2} \mathrm{Cu}_{3} \mathrm{O}_{7}$ и $\mathrm{La}_{2-x} \mathrm{Sr}_{x} \mathrm{CuO}_{4}$, определенные методом сравнения рассчитанных (в рамках модели точечных зарядов) и экспериментальных (методы ЭМС и ЯМР) параметров ядерного квадрупольного взаимодействия соответствуют стандартным степеням окисления атомов. Исключение составляют лишь атомы цепочечного $\mathrm{O}(1)$ и планарного $\mathrm{O}(3)$ кислородов в решетке $\mathrm{YBa}_{2} \mathrm{Cu}_{3} \mathrm{O}_{7}$ и атомы планарного $\mathrm{O}(2)$ кислорода в решетке $\mathrm{La}_{2-x} \mathrm{Sr}_{x} \mathrm{CuO}_{4}$, пониженный заряд которых объясняется локализацией в соответствующих подрешетках дырки. Подтверждение предложенных моделей пространственного распределения электронных дефектов в решетке $\mathrm{YBa}_{2} \mathrm{Cu}_{3} \mathrm{O}_{7}$ получено в результате анализа данных ЯКР на изотопе ${ }^{137} \mathrm{Ba}$, а в решетках $\mathrm{La}_{2-x} \mathrm{Sr}_{x} \mathrm{CuO}_{4}$ $(x=0.1-1.0)$ - в результате совместного анализа зависимостей $\left[V_{z z}\right]_{x} /\left[V_{z z}\right]_{x}=0.1$ и $\left.\left[e Q U_{z z}\right]_{x} /\left[e Q U_{z z}\right]_{x=0}\right)$ с использованием данных ЭМС на изотопах ${ }^{5} 7 \mathrm{Co}\left({ }^{57 m} \mathrm{Fe}\right)$, ${ }^{67} \mathrm{Cu}\left({ }^{67} \mathrm{Zn}\right),{ }^{67} \mathrm{Ga}\left({ }^{67} \mathrm{Zn}\right)$ и ${ }^{155} \mathrm{Eu}\left({ }^{155} \mathrm{Gd}\right)$. 


\section{Список литературы}

[1] К.В. Мицен, О.М. Иваненко. УФН. 187. 431 (2017).

[2] M. Takigawa, P.C. Hammel, R.H. Heffner, Z. Fisk , K.C. Ott, J.D. Thompson. Phys. Rev. Lett. 63, 1865 (1989).

[3] K. Ishida, Y. Kitaoka, G.J. Zheng. J. Phys. Soc. Jpn. 60. 1516 (1991).

[4] Y. Itoh, C. Michioka, K. Yoshimura, Y. Ueda. J. Magn. Magn. Mater. 310, 517 (2007).

[5] S.-H. Baek, A. Erb, B. Büchner. Phys. Rev. B 96, 094519 (2017).

[6] J. Shore, S. Yang, J. Haase, D. Schwartz, E. Oldfield. Phys. Rev. B 96, 595 (1992).

[7] N. Seregin, A. Marchenko, P. Seregin. Emission Mössbauer spectroscopy. Electron defects and Bose-condensation in crystal lattices of high-temperature supercomductors. Verlag: LAP Lambert. Academic Publishing GmbH\&Co. KG Saarbrücken (2015). 325 p.

[8] K. Schwarz, C. Ambrosch-Draxl, P. Blaha. Phys. Rev. B 42, 2051 (1990).

[9] J. Yu, A.J. Freeman, R. Podloucky, P. Herzig, P. Weinberger. Phys. Rev. B 43, 532 (1991).

[10] D.J. Singh, K. Schwarz, P. Blaha. Phys. Rev. B 46, 5849 (1992).

[11] Г.А. Бордовский, Е.И. Теруков, А.В. Марченко, П.П. Серегин. ФТТ 51, 2094 (2009).

[12] Г.А. Бордовский, А.В. Марченко, П.П. Серегин, Е.И. Теруков. Письма в ЖТФ 34, 79 (2008).

[13] K. Ivon, M. Francois. Z. Physik. B: Condens. Matter. 76, 413 (1989).

[14] A.F. Wells. Structural inorganic chemistry. Oxford (1984). $1120 \mathrm{p}$.

[15] M. Francois, A. Junod, K. Yvon, A. Kewat, J. Capponi, P. Strobel, M. Marezio, P. Fischer. Solid State Commun. 66, 1117 (1988).

[16] J.J. Capponi, C. Chaillout, A.W. Hewat, P. Lejay, M. Marezio, N. Nguyen, B. Raveau, J.L. Soubeyroux, J.L. Tholence, R. Tournier. Europhys. Lett. 3, 1301 (1987).

[17] J. Konstatntinovic, G. Parette, Z. Djordjevic, A. Menelle. Solid State Commun. 70, 163 (1989).

[18] Y. Le Page, T. Siegrist, S.A. Sunshine, L.F. Schneemeyer, D.W. Murphy, S.M. Zahurak, J.V. Waszczak, W.R. McKinnon, J.M. Tarascon, G.W. Hull, L.H. Greene. Phys. Rev. B 36, 3617 (1987).

[19] J.M. Tarascon, L.H. Greene. Science 236, 1373 (1987).

[20] A. Lombardi, M. Mali, J. Roos, D. Brinkmann. Phys. Rev. B 53, 14268 (1996).

[21] Г.А. Бордовский, Е.И. Теруков, А.В. Марченко, П.П. Серегин, А.В. Шалденкова. Письма в ЖТФ 43, 102 (2017).

[22] G. Wortmann, A. Kolodziejczyk, M. Bergold, G. Stadermann, C.T. Simmons, G. Kaindl. Mössbauer studies of $\mathrm{YBa}_{2} \mathrm{Cu}_{3} \mathrm{O}_{7-x}$ type high-Tc superconductors. Hyperfine Interact 50, 555 (1989).

Редактор К.В. Емиев 